\title{
Three-body problem, the measure of oscillating types. A short review
}

\author{
Christian Marchal \\ French National Office for Aerospace Studies and Researches (ONERA) \\ email:Christian.Marchal@onera.fr
}

\begin{abstract}
The theoretical three body problem, with three given non infinitesimal point masses, has two types of oscillating motions. In the first type at least two mutual distances are unbounded, but their inferior limit is bounded: there are an infinite number of larger and larger ejections, but without escape. In the second type, it is the velocities that are unbounded: there are an infinite number of nearer and nearer quasi-collisions, without exact collisions.

The first type has only a theoretical interest: its measure in phase space is zero. But the second type has a positive measure in phase space and a physical interest: it governs most of the collisions of stars.
\end{abstract}

Keywords. galaxies: evolution, three body problem, collisions

\section{The mechanism of indirect collisions}

Among the different types of three body motions there exists the exchange type: a single star meets a binary and their mutual gravitational interaction leads to the hyperbolic expulsion of one star of the binary and to the formation of a new binary with the two remaining stars. That exchange type is particularly frequent if, in the reference axis of the center of mass, the energy integral of the three body system of interest is negative.

Consider now our galactic system: a majority of stars are binary or multiple stars. If then a weak binary meets a strong binary the four body resulting motion is very close to a three body motion (the motion of the two stars of the weak binary and the center of mass of the strong binary) and motions of exchange type are certainly frequent. These exchange motions lead to the birth of new triple systems: the strong binary and one star of the weak one, these new triple systems that can sometimes be of the second oscillating type.

Of course motions of the second oscillating type lead real bodies to collision and thus the probability of collision through that indirect way is not negligible.

\section{The probability of collisions}

If we take account the interstellar distances, the average size of stars and their average velocities, the frequency of direct collisions of stars is extremely small: perhaps a few per million years in our galactic system. On the contrary, the formation (and the destruction) of new triple systems through the meeting of two binary or multiple systems is a frequent phenomenon, and thus we are interested into the probability of formation of triple systems having an oscillating motion of the second type.

The four presented references deal with that question that will be presented in the following way. We will use the usual Jacobi decomposition of the three-body problem into two two-body systems: the two nearest bodies are the inner system and the motion 
of the third mass with respect to the center of mass of the inner system is the outer system.

Let us call A the total angular momentum of the three-body system of interest, it is the vectorial sum of the angular momentums $\mathbf{A}_{\text {in }}$ and $\mathbf{A}_{\text {out }}$ of the inner and the outer systems. We will also call $\mathbf{A}, \mathbf{A}_{\text {in }}$ and $\mathbf{A}_{\text {out }}$ the corresponding moduli. The total angular momentum $\mathbf{A}$ is a well known integral of motion and the perturbations of the inner and outer systems over each other have generally little effect on the modulus $\mathbf{A}_{\text {out }}$ of the outer angular momentum (but the direction of that momentum can have large slow variations). Since the quasi-collisions of the two bodies of the inner system occur when its own angular momentum is very small, a usual condition for an oscillating motion of the second type is the almost equality of the moduli $\mathbf{A}$ and $\mathbf{A}_{\text {out }}$. This condition requires generally a very large mutual inclination of the inner and outer systems. For three given masses, it possible to have a rough estimate of the probability $P$ of an oscillating motion of the second type. Consider the inner and outer systems under the following conditions: the semi-major axes and the eccentricities are given (and then also the moduli $\mathbf{A}_{\text {in }}$ and $\mathbf{A}_{\text {out }}$ ) and the other parameters are free (with an isotropic repartition of the orientations). In these conditions there are two main cases:

I) If $\mathbf{A}_{\text {in }}>2 \mathbf{A}_{\text {out }}$, rare case requiring a small outer mass, in that case no orientation can give $\mathbf{A} \simeq \mathbf{A}_{\text {out }}$ and the probability $P$ is zero or very small.

II) If, as usual, $\mathbf{A}_{\text {in }}<2 \mathbf{A}_{\text {out }}$, the probability $P$ is generally a little larger than the ratio $\left(\mathrm{m}_{\text {out }} \mathrm{T}_{\text {in }} / \mathrm{M} \mathrm{T}_{\text {out }}\right)$, where $\mathrm{m}_{\text {out }}$ is the outer mass, $\mathrm{M}$ the total mass, $\mathrm{T}_{\text {in }}$ the period of the inner system and $\mathrm{T}_{\text {out }}$ that of the outer system.

Of course that ratio $\left(\mathrm{m}_{\text {out }} \mathrm{T}_{\mathrm{in}} / \mathrm{M} \mathrm{T}_{\text {out }}\right)$ is generally small, but it is far larger than those corresponding to direct collisions and the number of indirect collisions is perhaps one hundred, or even one thousand times larger than that of direct collisions. Notice that the energy of the collision of two average stars is about $10^{42}$ joules, which is much less than that of the supernova phenomenon, but this corresponds roughly to an ordinary nova phenomenon. It is then possible than the indirect collisions are responsible for a large proportion of the novae.

\section{Conclusion}

The importance, in phase space, of the oscillating motions of the second type may give them a major role in the evolution of the galactic system, even if the present theoretical study neglects many phenomenons as for instance the tidal effects that are certainly essential for bodies undergoing quasi-collisions.

\section{References}

Chenciner, A. \& Libre, J., 1988, Ergodic theorem and Dynamical systems, 8, 63-72

Fejoz, J., 2001 Journal of Differential Equations, 175, 175-187

Marchal, C., 1978 Acta Astronautica, 5, 745-764

Zhao, L., 2013, Thèse de Doctorat en Mathématiques Université Paris VII Diderot 